\title{
Josua Stylites und die damaligen kirchlichen Parteien des 0stens.
}

Abbé Martin im Vorwort zu seiner Ausgabe der Chronik des Styliten Josua (p. V) äufsert sich über dessen Glauben, wie folgt: „Quelle était sa croyance religieuse? Etait-il monophysite ou orthodoxe? Assémani, dans un but très-louable, a voulu le classer parmi les écrivains catholiques, mais, malgré son autorité, nous avons de la peine à nous ranger à son avis. Sans avoir aucun fait ou aucun texte précis à alléguer, nous croyons que Josué était monophysite. A cette époque, en effet, la Syrie chrétienne avait cessé a peu près toute entière, d'être orthodoxe." Ihm stimmt Alfred von Gutschmid bei (Kl. Schriften II S. 565): „In dem ganzen Buche kommt, was bei einem syrischen Mönche sehr anzuerkennen ist, nichts von theologischem Gezänk und keine Silbe von den zwei Naturen vor, was es Assemani möglich gemacht hat, ihn als Katholiken zu reklamieren; der Herausgeber hat vollkommen recht, dies im Hinblicke auf die damaligen kirchlichen Zustände Syriens für sehr unwahrscheinlich zu erklären und in Josua einen Monophysiten zu erkennen." Ebenso urteilt Th. Nöldeke (Z. D. M. G. XXX S. 352): „Was die konfessionelle Stellung Josuas betrifft, so urteilt Martin mit Recht, dafs man bei einem damaligen Edessener monophysitischen Glauben voraussetzen mufs, so lange man nicht starke Gründe für das Gegenteil hat."

Indessen die Sache ist keineswegs so klar, als es nach diesen Urteilen den Anschein hat. Schon der Satz Martins, dafs in Anastasius' Zeit fast das ganze christliche Syrien aufgehört habe, orthodox zu sein, bedarf gar sehr der Einschränkung. Mit Recht betont deshalb Nöldeke seine edessenische Abkunft; denn am ehesten mag diese Anschauung das Richtige treffen für die östlichen Kirchenprovinzen des Patriarchats Antiochien'), Osroëne und Mesopotamien. Immerhin möge man bedenken, dafs noch keine zwanzig Jahre seit der Schliefsung der per-

1) Natürlich sehe ich hier ganz von der Patriarchaldiöcese Jerusalem ab, wo aus Kyrillos von Skythopolis und der Zeitgenossen Mönchsviten die orthodoxe resp. nestorianisierende Richtung der Mönchskolonieen genügend bekannt ist. 
sischen Schule in Edessa verstrichen sind. Sollte ein so kurzer Zeitraum genügen, eine völlige Ausrottung der nestorianisierenden Richtung in der edessenischen Kirche zustande zu bringen? Das Kloster unsres Edesseners Zuknîn liegt allerdings in der eifrig monophysitischen Diöcese Amida $^{1}$ ); allein dafs auch unter dem dortigen Adel noch chalcedonensische Gesinnung vorhanden war, zeigt das Beispiel des comes Orientis und nachherigen Patriarchen Ephraïm, Appians Sohn. Xenaïas ferner beschuldigt die Mönche von Amida, dafs sie „den Eifer des Glaubens vernachlässigten" und vergleicht sie mit dem Verräter Judas, was, wie bereits Assemani (B. O. II 37) gewifs richtig erklärt hat, auf geringen Eifer für die monophysitische Sache deutet. Dafs aber in der Euphratensis und speziell in Hierapolis die Synoditen sehr einflufsreich waren, ja zeitweise die Oberhand hatten, bezeugt für die Basiliskos- und Zeno-

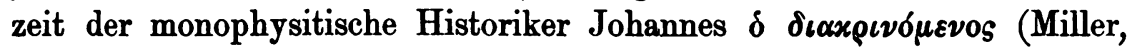

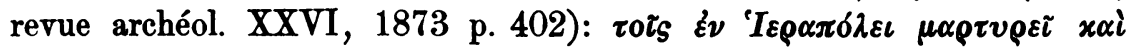

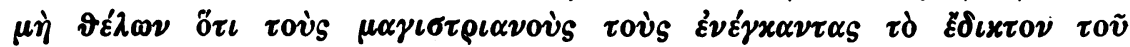

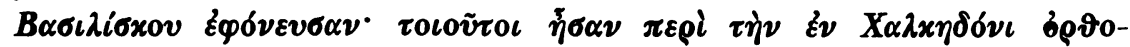

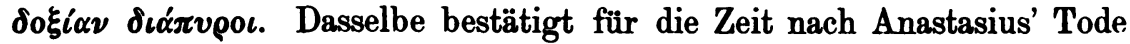
ein gewils vollgültiger Zeuge, Xenaïas von Mabbôg selbst, welcher diese Gesinnung in den von Assemani (B. O. II 44) publizierten Auszügen bitter beklagt: „Während überall viele als würdige Bekenner für Christus aufgetreten sind, hat diese Stadt, deren geistliche Leitung mir anvertraut war, sich dieses Gutes unwürdig gezeigt, damit ich nicht das Gegenteil sage. Denn etliche unter ihnen sollen lieber die Zahl der Verfolger als der Verfolgung Leidenden haben vermehren wollen." Ferner: „Nun aber, uneingedenk ihrer Thaten, schreiben sie an den Usurpator des antiochenischen Stuhles (Paulus), wie mir gemeldet worden ist, sie seien die ganze Zeit, wo wir als Hirten ihre Leitung hatten, in Finsternis gehüllt gewesen; jetzt aber, nachdem sie die Synode anerkannt hätten, seien sie zum Lichte durchgedrungen." Die thätlichen Anfeindungen, welche, wie er klagt, ihn sowohl in seiner Metropolis, als im westlichen Teil seines Sprengels, in der Kyrrestike, betroffen haben, zeigen klar, dafs zum mindesten der Erzbischof mit einer sehr starken synoditischen Minorität zu rechnen hatte. Vollends in deu westlichen Eparchieen der antiochenischen Diöcese blieben zahlreiche Bischöfe, Klöster und Gemeinden, nachdem Severus von Anastasius zum Patriarchen eingesetzt worden war, in ständiger Opposition. Euagrios (III 33) gedenkt in Phönizien der Bischöfe von Tyros und Berytos,

1) cfr. Assemani B. O. I p. 260. Joannis Ephesi de beatis Oriental. verterunt van Douwen et Land p. 111 u. 130. 
im libanensischen Phönizien des Bischofs von Damaskos und in Arabien des von Bostra. Besonders Syria II zeigte eine scharfe antimonophysitische Richtung. Die Bischöfe von Epiphaneia und Arethusa finden dabei einen starken Anhalt an der Bürgerschaft ihrer Städte ${ }^{1}$ ), weshalb Anastasius in sehr verständiger Weise von jeder gewaltthätigen Mafsregel Abstand zu nehmen gebietet. Als Xenaïas mittelst der Mönchsscharen von Syria I eine geistliche Revolution gegen Flavian von Antiochien zu inscenieren versucht, werfen die Antiochener die Mönche in den Orontes, und die Mönche von Syria II, unter denen Flavian einst als Ascet geweilt hatte, eilen als seine Leibgarde nach der Hauptstadt. $\left.{ }^{2}\right)$

Auf die den Monophysiten höchst feindseligen Eingaben der orientalischen Mönche an die Synoden unter Justin I. und Justinian mit ihren zahlreichen syrischen Unterschriften wird man schwerlich viel geben können. Denn man sieht zu deutlich, dafs man hier bestellte Arbeit vor sich hat. Immerhin mögen einige der thatsächlichen Mitteilungen, wenn sie auch stark gefärbt sind, nicht geradezu erfunden sein. In Tyros, dessen Erzbischof, der Protothronos von Antiochien, dem Severus sehr hartnäckig widerstanden hatte, soll dieser die anfänglich zu ihrem Ordinarius haltenden, nachher freilich sich fügenden Presbyter zu Diakonen degradiert haben. Ähnliche Gewaltthätigkeiten werden aus den Diöcesen Arke, Tripolis, Antarados gemeldet (Mansi VIII 1075 sq.). Aus den Berichten über die zahlreichen Verfolgungen der Orthodoxen in Phönizien geht jedenfalls hervor, dafs auch unter Severus ihre Zahl nicht unbeträchtlich war. Aus den inhaltlich wenig erheblichen Akten gegen Petros von Apameia ergiebt sich wenigstens, dafs die Lektoren seiner Kathedralkirche nicht zu seiner Partei hielten (Mansi VIII 1107 sq.). Das Bisherige, so fragmentarisch es ist, mag immerhin beweisen, dal's von einem Aufhören des orthodoxen Bekenntnisses in Syrien für diese Zeit noch nicht gesprochen werden kann.

Was nun Josua speziell betrifft, so sind wir inbetreff seiner Glaubensrichtung auf die eigenen Aussagen desselben über die gleichzeitigen Bischöfe angewiesen. Die Bischöfe Stratonikos von Karrae und Barhadad von Konstantine-Tellâ, Thomas und Nonnos von Amida, von

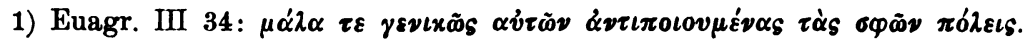

2) Jakob von Sarûg im Briefe an die Mönche des Klosters von Mar Bassus bemerkt, dafs im Gegensatze zu Ägypten gerade Syrien das Chalcedonense annahm ,à cause de l'Archevêque Jean d'Antioche, Jean, qui avait partagé les idées de l'impie Nestorius." Z. D. M. G. XXX p. 263. Fast möchte man annehmen, dafs Jakob den Johannes von Antiochien noch als lebend zur Zeit des Chalcedonense ansah. Jedenfalls kann dies Zeugnis nicht sehr ins Gewicht fallen. 
denen die beiden erstern wahrscheinlich, die letztern sicher Monophysiten waren, erwähnt er so, dafs seine Bemerkungen nach keiner Seite hin entscheiden. Dagegen werden mehrere Monophysiten mit augenscheinlicher Hochachtung behandelt, so Jakob von Batnae (Wright S. 43), der damals freilich erst Periodeut war, und vor allem die beiden Bischöfe von Edessa: Kyros und Petros. Nach Assemanis (B. O. I 292) wenig wahrscheinlicher Behauptung ist letzterer ein Orthodoxer, während Kyros ganz sicher Monophysit war. Josua jedenfalls lobt den religiösen Eifer des einen wie des andern (the chronicle of Joshua the stylite by W. Wright p. 19, 23, 27, 29). Daneben vergleicht er auch den palästinensischen, also höchst wahrscheinlich orthodoxen Bischof von Nikopolis, der allein mit seinen beiden Syncellen dem Erdbeben entrann, „dem gerechten Lot, als er aus Sodom entkam" (Wright p. 25). Von Wichtigkeit sind allein die Aussagen über Xenaïas und Flavian, welche letztere schon Assemani ins gebührende Licht gestellt hat. Bei Anlafs des Todes des Bischofs Johann von Amida sagt Josua (Wright p. 66): „Und sein Klerus kam zu dem heiligen und gottliebenden, mit allen göttlichen Schönheiten geschmückten, trefflichen und erlauchten Mâr Flavian, Patriarchen von Antiochien, um ihn zu bitten, ihnen einen Bischof einzusetzen." Dagegen des Xenaïas gedenkt .er bei der Wiederkehr des oft von ihm getadelten und beklagten „heidnischen“ Festes (p. 21): „Aber obwohl Xenaïas, der Bischof von Mabbôg, zu dieser Zeit in Edessa war, von dem man eher als jedem andern denken konnte, dafs er die Mühe des Unterrichts auf sich genommen hätte, sprach er mit ihnen (den Edessenern) nur einen einzigen Tag über diesen Gegenstand." Das warme und sehr wortreiche Lob Flavians ist in der Chronik ganz singulär; keiner der zahlreich erwähnten Kirchenfürsten wird von dem Annalisten irgend ähnlich ausgezeichnet. Die hohe Stellung des Patriarchen erklärt das allein nicht; denn er war in seiner Diöcese starken Anfeindungen ausgesetzt. Um so mehr sticht dagegen die recht kühle Behandlung des Xenaïas ab, welche, wenn auch in zurückhaltender Weise und in bescheidenen Ausdrücken, den Tadel der Menschenfurcht ausspricht. Xenaïas als kluger Diplomat hielt es offenbar für angezeigt, gegenüber der im Dogma so korrekten Bürgerschaft von Edessa im Punkte der Moral etwas weitherzig zu sein; solche schlaue Parteitaktik war nun freilich nicht nach dem Sinne des aufrichtigen Josua, dem seine strenge Mönchsmoral entschiedene Herzensüberzeugung war. Es kommt hinzu, dals Flavian und Xenaïas erbitterte Feinde waren; wer aber dem Flavian so hohe Verehrung bezeugt, kann unmöglich ein korrekter Monophysit in der Art des Severus und Xenaïas sein. 
Die damaligen kirchlichen Verhältnisse Ostroms und besonders Syriens waren so verwirrt ’als möglich. Wenn die palästinensischen Mönche an Alkison schreiben (Euagr. III 31), dals die Zahl der prinzipiellen Dioskorianer sehr zusammengeschwunden sei, so zeigt der $\mathrm{Zu}$ sammenhang, in dem die Worte stehen, deutlich, dafs allein vom Osten, d. h. der Diöcese Antiochien die Rede ist. Dieselben Mönche sagen unmittelbar vorher, dafs gegenüber dem chalcedonensischen Westen und dem mehr vermittelnden Osten Agypten und Alexandria eine vollkommene Sonderstellung einnahm, d. h. hier herrschten die entschiedenen Monophysiten, welche auch stets den Rückhalt für ihre Gesinnungsgenossen in Syrien und Mesopotamien bildeten. Die beiden zeitgenössischen

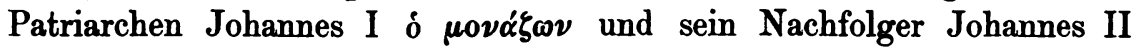

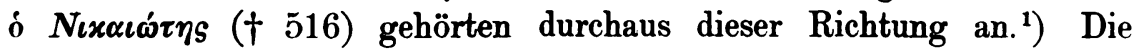
monophysitischen Berichte betonen, dafs sie dabei mit der Zentralregierung stets im besten Einvernehmen lebten. Bei der bedeutenden Stellung, welche das damalige alexandrinische Patriarchat einnahm, lohnt es sich, die Lebensbeschreibungen der beiden aus der noch nicht veröffentlichten Hälfte des koptisch-arabischen Synaxars, welche ich der Gefälligkeit des Herrn Professor Wüstenfeld in Göttingen verdanke, hier zu veröffentlichen.

\section{Tag des Monats Baschnas (- Pachon, 29. April).}

An diesem Tage verschied der heil. Vater Anba Johannâ, Patriarch von Alexandria. Dieser Vater war ein Kind aus den gläubigen Einwohnern von Alexandria und widmete sich von Jugend auf dem Mönchsleben in dem Askit des Abu Makarios. Nach dem Tode des Vaters Athanasios wurde er durch den Willen der sämtlichen Bischöfe und Gelehrten zum Oberhaupte gewählt, und er nahm die Stelle mit Widerstreben an, da er sie nicht wünschte, sondern erst durch vieles Bitten bewogen wurde, es keinem andern zu überlassen an der Spitze des Volkes zu stehen und es zu leiten. Als er sah, wie die Bischöfe und Altesten ihn baten, gab er ihnen demütig nach, indem er sagte: „Vielleicht ist es der Wille des Messias." Sobald er sich auf den Thron gesetzt hatte, sorgte er für das Volk im höchsten Grade durch Unterweisung, Vorlesen und Stärkung im festen heiligen Glauben und ging darin den Bischöfen und gelehrten Priestern voran. Der damalige Herrscher Zeno der Fromme war ein heiliger Gläubiger, welcher sich mit

1) In Alexandria herrschte diese Richtung von Anfang an. Ganz richtig leitet Johannes von Nikîu die Unruhen unter Petros Mongos daher, weil dieser das Henotikon acceptierte, während Volk und Klerus an der ausdrücklichen Verdammung des Chalcedonense festhielten. Notices et extraits XXIV 1 S. 483. 
diesem Heiligen eng verband und seine Hand über das Land ausbreitete, so dafs der feste Glaube in allen Gebieten von Ägypten öffentlich verkündigt wurde. Der Herrscher schickte in jenen Tagen in die Wüste des Makarios Ladungen von Getreide, Wein und Öl, um davon das, was sie zum Lebensunterhalt nötig hatten, zu bestreiten. Die ganze Zeit dieses Vaters verlief in Ruhe und Frieden, und Gott segnete die Menschen durch das Gebet dieses Vaters und durch seine Unterweisung. Dann suchte ihn der Herr heim durch eine kurze Krankheit und nahm ihn zu sich, nachdem er acht Jahre auf dem Throne gesessen hatte. Sein Gebet sei mit uns, Amen!

\section{Tag des Monats Baschnas (= Pachon, 22. Mai).}

An diesem Tage ging zur seligen Ruhe der heil. Vater Patriarch Anba Johannâ. Dieser Heilige war in seinem Glauben und Wandel ein christlicher Mann, welcher sich schon von seiner Jugend an dem Mönchsleben gewidmet und sich selbst in jeder Art des heil. Kampfes geübt hatte und sich dann selbst in ein Kloster einschlofs. Der Ruf seiner Gelehrsamkeit und Frömmigkeit verbreitete sich, und er wurde für das Patriarchat in der Stadt Alexandria gewählt. Er schrieb in seinen Tagen viele Verordnungen, und Gott richtete zur Zeit dieses Vaters die Säule der Kirche auf, weil der gläubige, gottesfürchtige Anastasius Herrscher und der Vater Anba Severus Patriarch auf dem Throne von Antiochia war. Da erliefs der heil. Severus ein Synodalschreiben an diesen Vater Johannâ über die Übereinstimmung im Glauben und setzte ihm darin auseinander: „Siehe, Christus ist unser Gott, nachdem er eine einzige eigene Natur ohne Teilung in sich vereinigt hat, und wir sind des Glaubens des Vaters Kyrillos und des Vaters Dioskoros." Dies nahm der Vater Johannâ mit seinen Bischöfen an, und sie liefsen Dank- und Lobgebete zu Gott aufsteigen für die Wiedervereinigung der getrennten Glieder an ihrer Stelle. Dann schrieb ihm der Vater Johannâ als Antwort auf seinen Brief mit Worten voll aufrichtigen Dankes, womit er die Einheit des Wesens Gottes, die Dreifaltigkeit seiner Person, die Verkörperung des Sohnes von Ewigkeit in der menschlichen Natur bezeugte, und dafs beide durch die Vereinigung einer, nicht zwei geworden seien. „Fluch dem, welcher Christus teilen oder seine Natur vermischen will, und allen denen, die da sagen, dafs der, welcher gelitten hat, gekreuzigt worden und für die Menschheit gestorben ist, ein einfacher Mensch gewesen sei oder die Schmerzen und den Tod nach der Natur einer Gottheit erlitíen habe; vielmehr isí der festsíehencie Glaube, dafs wir bekennen, dals Gott das Wort für uns gelitten habe in dem Körper, in welchem er mit uns eins geworden ist, und dies ist 
der königliche Weg, welcher den nicht irre führt und straucheln macht, der auf ihm wandelt." - Als der Vater Severus dieses Schreiben gelesen hatte, nahm er es wohlgefällig auf und verkündete es von dem Throne in Antiochia, und die Einigkeit und Übereinstimmung zwischen beiden blieb eine beständige. Dieser Vater blieb als Prediger und Hüter seiner Gemeinde die Zeit von elf Jahren, dann ging er in Frieden zur Ruhe. Sein Gebet und seine Vermittlung sei mit uns, Amen!

Ein Vergleich dieser Angaben mit den übrigen koptischen Berichten, vorab mit Ibn Rahib (chron. or. p. 99) und der von Renaudot (hist. patr. Alex. p. 125 ff.) gegebenen Übersetzung der Angaben des Severus von Ašmunîn und dem von demselben gefertigten kurzen Auszuge aus dem ersten unveröffentlichten Teile des Elmakîn zeigen, dafs der Bericht des Synaxars auf diese Quellen zurückgeht. Ob er freilich die Auszüge aus den Briefen des Severus und des Johannes aus Elmakîn hat, lärst sich bei der Knappheit von Renaudots Aussage nicht mit Sicherheit feststellen. Auffällig ist, dafs alle diese Berichte den Johannes I. zum Zeitgenossen Zenos machen. Das stimmt nicht mit der Chronologie; Le Quien setzt ihn 496-507 und Gutschmid 496-505, also unter Anastasius. Ausdrücklich erwähnt auch Liberatus (breviar. 18) noch Johanns Vorgänger Athanasios als Zeitgenossen des Anastasius. Obschon auch eine von diesen koptischen Berichten durchaus unab-

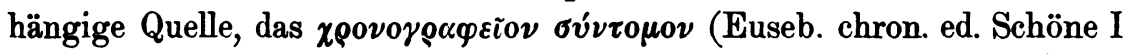

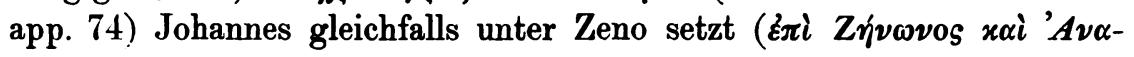
$\sigma \tau \alpha \sigma i o v)$, scheint doch hier ein allerdings recht alter Fehler vorzuliegen; denn die Chronologie auch der vorangehenden Patriarchen schliefst jede Gleichzeitigkeit von Johannes und Zeno gebieterisch aus. ${ }^{1}$ )

Über die gleichzeitigen syrischen Verhältnisse giebt am besten Euagrios III 30 Auskunft, welcher dieselben nicht ohne Ironie schildert $^{2}$ ), aber zugleich mit einer für einen orthodoxen Schriftsteller anerkennenswerten Objektivität die Tendenz von Anastasius' Kirchenpolitik in dessen früheren Jahren klarstellt. Er unterscheidet drei Richtungen unter den damaligen Kirchenfürsten. Die einen hielten mit der gröfsten Entschiedenheit an den Beschlüssen von Chalcedon fest, ohne auch

1) Vielleicht wird die Erklärung durch die Angabe des Synaxars gegeben, wonach Johannes früher Mönch des Makariosklosters gewesen war. Die Schenkung Zenos wird in die Zeit gefallen sein, wo er noch Mönch war, und ist dann irrtümlich in die Epoche seines Patriarchats verlegt worden.

2) Durch den 150jährigen Streit über die zwei Naturen waren in Mauricius' Tagen die Gebildeten in Syrien (Euagrios war dazu Jurist) vollkommen indifferent geworden. Es ist aber nicht richtig, wenn man deshalb in Euagrios einen verkappten Heiden hat sehen wollen. 
nur in einem Buchstaben nachzugeben; vielmehr kündigten sie jedem die Kirchengemeinschaft, der das Chalcedonense nicht annahm. Andere dagegen verwarfen nicht blofs das Chalcedonense, sondern sprachen über seine Definitionen und Leos Tomos das Anathem aus. Endlich die dritten hielten sich an das Henotikon Zenos hauptsächlich aus Liebe zum Frieden; indessen auch diese Henotiker zerfielen in chalcedonensisch und mehr monophysitisch Gesinnte. Anastasius verfolgte die Politik, alle Richtungen möglichst gewähren zu lassen; an jedem Orte sollte die in den letzten Dezennien ausgebildete Tradition mafsgebend sein. Nur wo ein Kirchenfürst einen dem örtlichen Herkommen widersprechenden Standpunkt einnahm, schritt er mit Absetzungen ein, um die Ruhe herzustellen.

Vor allem ist nun wichtig, die dogmatische Stellung des Flavian möglichst genau zu präzisieren, was nicht ganz ohne Schwierigkeit ist. Johannes von Nikiû (l. c. p. 497) lälst die orientalischen Bischöfe in Byzanz Klage führen, dafs Flavian trotz seiner Annahme des Henotikons verkappter Nestorianer sei und das Chalcedonense, wie Leos Tomos acceptiert habe. Ebenso sagt Johannes von Ephesos (I 41), dafs er der Häresie der zwei Naturen überführt worden sei. So einfach liegt die Sache keineswegs. Die palästinensischen Mönche in ihrem Briefe an Alkison und Theophanes, welcher dem Theodorus Lector folgt, erzählen, dafs er, eingeschüchtert durch Xenaïas und die korrekt monophysitischen Bischöfe, sich allmählich immer entschiedener monophysitisch gefärbte Glaubensbekenntnisse habe abdringen lassen.

Theophanes berichtet (S. 151, 11, vgl. den Parallelbericht bei Euagr. III 31), dafs er auf Befehl des Kaisers, - nach dem Bericht der Mönche auf Instigation des Xenaïas - 508/9 eine Synode (wohl in Antiochien) versammelte, und deren Beschlüsse dem Kaiser in einem nusführlichen Schreiben mitteilte. Darin bekannte er sich, getreu dem Henotikon folgend, zu den Synoden von Nikäa, Konstantinopel und Ephesos; dagegen das Chalcedonense überging er mit Stillschweigen. Aufserdem hat er ïber Diodor und Theodor (und nach den Mönchen auf Verlangen des Xenaïas schliefslich auch über zahlreiche angebliche oder wirkliche Gesinnungsgenossen derselben) das Anathem ausgesprochen und zum Schlusse vier Sätze $(\varkappa \varepsilon \varphi \alpha ́ \lambda \alpha \iota \alpha)$ verkündigt, welche der Lehre von Chal-

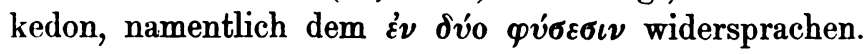

Indessen auch damit begnügte sich Xenaïas nicht; er verlangte eine ausdrückliche Verdammung des Chalcedonense; allein der Patriarch willfahrte nur in Bezug auf die diphysitische Glaubensdefinition; dagegen die daselbst vollzogene Verurteilung des Nestorios und des Eutyches billigte er ausdrücklich. Man sollte meinen, damit hätte er allen billigen 
Anforderungen genügt; aber nichtsdestoweniger betrachteten ihn fortan die Strengen als Kryptonestorianer. Umgekehrt kündigte ihm auch Makedonios in Konstantinopel die Gemeinschaft. ${ }^{1}$ )

Xenaïas suchte nun durch eine feierliche Provinzialsynode des Ostens Flavian zu diesem letzten Schritte moralisch zu zwingen. Auf Betrieb des Xenaïas und auf Befehl des Kaisers wurde denn die Synode offenbar sehr wider Flavians Wunsch nach Sidon berufen. Über diese besitzen wir einen ausführlichen Bericht in der syrischen Kirchengeschichte des sog. Zacharias von Mitylene, welche in erwünschter Weise die Angaben des Kyrillos in der vita des heil. Sabas, des Marcellinus comes und des Theophanes ergänzt. ${ }^{2}$ )

Es heifst da im X. Kap. des VII. Buches: „Aber auch über Flavian schrieb er (Xenaïas) an Anastasius, dafs er ein Häretiker sei, und riet deshalb die Abhaltung einer Synode in Sidon an. Und er befahl, und sie versammelte sich in der Zahl der Antiochener $560(=511 / 12)$. Und er instruierte die gläubigen und eifrigen Mönche des Ostens und den Kosmas, einen beredten Mann aus dem Kloster des Mar Akiba von Kinnesrîn (Chalkis), welcher in Antiochien wohnte. Und er machte eine Thesis und produzierte sie vor Flavian und der Versammlung der Bischöfe, welche mit ihm in Sidon waren, weislich und konsequent, Beschuldigungen in 77 Sätzen und viele $\chi \varrho \eta \sigma \varepsilon \iota \varsigma$ der heil. Lehrer, welche bewahrheiteten die Anklagen gegen die Synode von Chalcedon und den Tomos des Leo. Er liefs (es) schreiben und gab (es) an die Synode. Sie überzeugten die Priester und liefsen sie schwören, dafs sie Berichtigungen machten und abthäten die AnstöIse gegen die Gebräuche der Kirche, und sie reinigten sie, indem sie öffentlich die Synode verdammten. Flavianus aber, welcher das Haupt der Priester war, und die Anhänger desselben unter den Priestern hinderten ihn an der Ausführung, indem sie sagten: „Es genügt uns, dafs wir die Schrift der Partei des Diodoros bannen und die Widerlegungen, welche waren von den Leuten gegen die zwölf Kapitel des Kyrillos und von (für?) Nestorios, auf dafs wir nicht aufwecken den untern Teil, der schläft, und durch sein Gift schädigen die Menge. Solchermafsen wurde die Synode beendigt."

Wie man sieht, ist sie ein vollkommener Mifserfolg des Xenaïas; damit stimmt der Bericht Kyrills im Leben des heil. Sabas überein, welcher dies Resultat dem energischen Eintreten des gleichfalls anwesen-

1) Die Synoditen strikter Observanz hatten ihn schon bei seinem Regierungsantritt als verdächtig angesehen. (Theophan. 142, 11.)

2) Ich verdanke die Utbersetzung von Land Anecdota III S. 225 u. 228 der erprobten Gefälligkeit meines verehrten Kollegen Stickel. 
den Elias von Jerusalem für Flavian zuschreibt (Cotelerius eccles. gr. monum. III p. 301). Flavian lehrte wie die Monophysiten; aber eine Verdammung des Diodoros, Theodoros und Nestorios genügte ihm; eine ausdrückliche Verurteilung des Chalcedonense - und das war Xenaïas' Ziel - hielt er für überflüssig und gefährlich. Schliefslich soll er doch noch, bedrängt durch die vom Kaiser aufgehetzten Mönche, auch das Anathem über Chalcedon ausgesprochen haben (Theophanes 153,29ff.). Übrigens halfen ihm alle diese Konzessionen nichts; er ward abgesetzt,

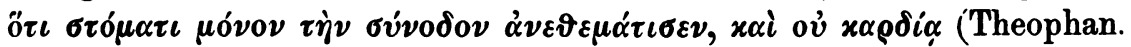
156, 12). Damit hat er übrigens seine Reputation als Sanctus gerettet ${ }^{1}$ ); die Fragmente aus seiner Homilie über Johannes V 23 und die Himmelfahrt (Mai: Script. vet. nova coll. VI 135) lauten in der That korrekt diphysitisch, und werden deshalb auch von Leontios in seiner Streitschrift gegen die Monophysiten unter die Zeugnisse unserer auserwählten Väter mit aufgenommen.

Flavians Brief an Anastasius und ebenso seine Taktik auf dem Konzil von Sidon entsprechen so ziemlich dem später zu erwähnenden ersten Briefe des Jakob von Sarûg an die Mönche von Mar Bassus, worin dieser Diodoros, Theodoros und Theodoret verdammt. Man sieht, es ist System in diesem Vorgehen; die Verdammung des Nestorios genügte nicht, auch alle Häupter der antiochenischen Schule und die nestorianisierenden Väter ${ }^{2}$ ) mufsten mit verdammt werden.

Was ist nun das ursprüngliche Bekenntnis Flavians? Offenbar gehörte er zu der von Euagrios geschilderten dritten Gruppe der wahren Henotiker im Sinne des Akakios, welche sich stricte an das Unionsedikt hielten und über die alles spaltende Frage der einen oder der zwei Naturen eine sehr verständige Zurückhaltung beobachteten. (Unter Umständen nennen das auch die Heiligen „eine kluge Ökonomie zur Rettung vieler Seelen“.) Um es kurz zu sagen, Flavian gehörte zu den

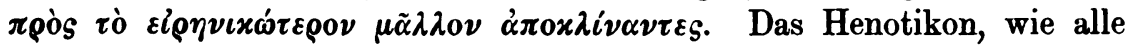
Vermittlungsversuche, konnte es den Eiferern beider Parteien nicht recht machen. Damit stimmt, dafs sowohl der streng monophysitische Alexandriner Johannes II., als das in seinen Vorstehern vor Timotheos gut synoditische Konstantinopel Flavian die Gemeinschaft kündeten. Mit der Annahme der ersten Forderung des Xenaïas, der Verdammung der

1) Baronius hat von seinem Standpunkte aus ganz recht, wenn er die Heiligkeit Flavians und des Elias von Jerusalem verwirft; und Tillemonts Proteste sind zwar gut gemeint, treffen aber neben das Ziel.

2) Ein ähnliches Verzeichnis giebt Victor Tunnunensis bei der Aufzählung der auf dem konstantinopolitanischen Konzil im Jahre des Johannes Gibbus (499) Verdammten. 
nestorianisierenden Väter, hat Flavian kein Opfer weder seines Intellektes, noch seines Gewissens gebracht. Diese Unionsfreunde des Ostens waren allezeit streng antinestorianisch. Die Ironie des Schicksals wollte es, dafs, was sie damals als ihre Konfession formulierten: Festhalten an den drei ersten Konzilien und Verdammung des Theodoros, Theodoret u. s. f. im schneidendsten Gegensatz zu den Beschlüssen von Chalcedon und zur gerechten Entrüstung des korrekt diphysitischen Abendlandes Justinian auf dem fünften Konzil zur orthodoxen Lehre erhob. So ehrfürchtig man dort auch im allgemeinen von Chalcedon sprach, thatsächlich hat man dasselbe in der Hauptsache eskamotiert; auch darin trifft die spätere Orthodoxie mit diesen Vermittlern zusammen, dafs beide gleichmäfsig einer unbedingten und vollständigen Verdammung des Chalcedonense bis zum äufsersten sich widersetzten.

Es war nun ein überaus verhängnisvoller Fehler des Anastasius, dafs er jene so überaus nützlichen Männer der Mittelpartei nicht halten konnte oder wollte. Dem Reiche wären ohne den jetzt eintretenden Umschwung die verhängnisvollen Wirren der nachfolgenden fünfzig Jahre erspart worden. Aber dije byzantinische Regierung hat in den so zart anzufassenden kirchlichen Dingen oft eine recht unglückliche Hand gehabt. Der Kaiser liefs sich von fanatischen Ratgebern (Marinos von Apameia) beeinflussen. Er war alt und abgenutzt und offenbar mehr geschoben, als selbständig handelnd, als er 512 sich zu einem entschiedenen Systemwechsel entschlofs und die Mittelpartei den Extremen opferte. Flavian ward exiliert, und an seine Stelle trat das Haupt der strengen Monophysiten, Severus, fragelos die bedeutendste theologische Kapazität dieser Epoche. Jetzt endlich wurden auch die langjährigen Bemühungen des Xenaïas mit Erfolg gekrönt. Was Flavians Autorität noch in Sidon verhindert hatte, wurde unter Severus mit Glanz durchgesetzt. Auf einer grofsen Synode der orientalischen Bischöfe zu Tyros wurde das Chalcedonense feierlich verdammt. Über diese Synode hatten wir bisher nur den Bericht des Dionysius von Tell Mahrê (Assemani B. O. II 19), welcher aber, wie schon Assemani bemerkt, eine falsche Zeitangabe hat (J. d. Seleuciden $826=515$ ). Sie kann nicht später als 5̃13 fallen, da Elias von Jerusalem noch im Amte ist. Der Bericht ist auch völlig unhistorisch; er erwähnt die Anwesenheit von Vikarien der Erzbischöfe von Konstantinopel und Jerusalem und des Erzbischofs von Alexandrien, ja sogar des Papstes Symmachus. Man sieht deutlich die Absicht der Spätern, dem orientalischen Diöcesankonzil ökumenischen Charakter zu verleihen. Auch soll das Henotikon verflucht worden sein, was keineswegs der Fall war; es wurde nur "richtig“ interpretiert. Den Bericht über die wahren 
Vorgänge verdanken wir wiederum der syrischen Kirchengeschichte (Land, Anecdota III p. 228):

„Zwölftes Kapitel über die Synode in Tyros, Kundmachung in den Tagen des Severus und des Xenaïas, der Lehrer und Bischöfe, die mit ihm waren und deutlich und öffentlich die Synode und den Tomos verdammten.

Severus aber, der nach Flavian in Antiochia war, war ein Mann durch das Lesen der Weisheit der Griechen beredt und freiwillig arm, erprobter Mönch, auch eifrig im wahren Glauben und bewandert, und las mit Verständnis auch in den heil. Schriften und deren Auslegungen, von den alten Aufzeichnungen der Schüler der Apostel: Hierotheos und Dionysios ${ }^{1}$ ) und Titus, auch Timotheos und derer nach ihnen, Ignatios und Clemens und Irenäus und der Anhänger des Gregorios, Basileios und Athanasios und des Julius und der übrigen Häupter der Priester und rechtgläubigen Lehrer der heiligen Kirche, und wie die Schrift, die gelehrt ward für das Himmelreich, hervorgegangen aus den alten und neuen Symbolen. Solchermafsen und durch viele Mitteilungen unterrichtete er sich, fest gegründet in seiner Überzeugung von klarer Einsicht.

Und jener Xenaïas war auch ein syrischer Lehrer und bewandert in dem, was in dieser Sprache vorhanden ist. Auch er beschäftigte sich mit Fleifs mit ihnen, auch in der Lehre der Anhänger des Diodoros und Theodoros und der übrigen war er bewandert. Wie nun diese Gottesverehrer lehrten die getrennten Gläubigen, so war jener ehrwürdige und eifrige Mann für die Wahrheiten. Solches that man kund dem Kaiser Anastasius, der aus voller Überzeugung das Konzilium von Chalcedon ausdrücklich verdammte. Er verordnete, dafs zur Berichtigung dessen, was verlangt würde, eine Synode der Orientalen in Tyros versammelt werde. Und sie versammelten sich von Bischöfen aus der Gegend von Antiochia und Apameia und Euphratensis und Assyrien und Arabien und Phönizien am Libanos, und so war der Osten für den wahren Glauben. Und er (Xenaïas) erläuterte seine Schrift über das Henotikon Zenos, welches zur Beseitigung dessen diente, was in Chalcedon festgestellt worden war. Und daselbst verdammten sie öffentlich die Zusätze, die zum Glaubensbekenntnis gemacht worden waren. Und die Bischöfe, welche mit Severus und Xenaïas versammelt waren, pro-

1) Die Stelle ist interessant als eines der ältesten Zeugnisse für die Schriften des Dionysius Areopagita. Noch älter ist das bei Liberatus im breviarium X, wonach schon Kyrillos in seinen vier Büchern gegen Diodoros und Theodoros ihu unter den Vïtern citierte. Freilich bestritten die Orthodoxen Kyrills Autorschaft; dem fünften Jahrhundert wird aber das Werk gleichfalls angehören. 
mulgierten die volle Wahrheit. Gläubige Männer und Lehrer, welche an der Spitze der Bischöfe waren, traten eifrig auf und schrieben Briefe der Beistimmung auch an Johannes von Alexandria und an Timotheus in der Residenzstadt. Auch Elias, zu dieser Zeit in Jerusalem, stimmte ihnen bei. Nach kurzer Zeit wurde er abgesetzt, und Johann folgte ihm. Solchermafsen vereinigten sich die Priester aufser dem Stuhl der Römischen über diese Glaubensunion."

Ich brauche nicht des nähern auszuführen, wie sehr dieser Bericht gegenüber dem des Dionysius von Tell-Mahrê den Vorzug verdient.

Ich glaube nun nicht zu irren, wenn ich der vermittelnden Gruppe der Friedensfreunde im Sinne Flavians, welche in Tyros so entschieden zurückgewiesen werden, auch den Styliten Josua beizähle. So erklärt sich am besten seine begeisterte Verehrung für den irenischen Flavian und seine nicht undeutliche Abneigung gegen den entschiedenen und fanatischen Xenaïas. Dieser Standpunkt, wenn wir so sagen dürfen des Kryptomonophysitismus, erklärt auch seine für die damalige Zeit aufsergewöhnliche Zurückhaltung in dogmaticis; er wollte mit seiner Chronik nach keiner von beiden Seiten Anstofs erregen.

Dadurch erhalten auch die sonderbaren Worte des 101. Kapitels (Wright p. 76) die richtige.Beleuchtung: „Wenn dieser Kaiser gegen das Ende seines Lebens in einem andern Lichte erscheint, so soll sich niemand an diesen Lobpreisungen (die im Texte vorangehen) stofsen, sondern dessen gedenken, was Salomo am Ende seines Lebens that." Mit Berücksichtigung dieser Stelle nimmt von Gutschmid (Kl. Schriften II S. 566) an, dafs die Chronik zwar unter dem frischen Eindrucke der Ereignisse, also wohl noch 507 verfafst, aber erst nach dem Tode des Anastasius 518 veröffentlicht worden sei. Wright (preface S. IX) dagegen verlegt die Abfassung in den Winter 506 und den Anfang des folgenden Jahres, Nöldeke (Z. D. M. G. XXX, 1876 S. 352) bald nach November 506. Beide betrachten das Urteil über Anastasius als einen spätern Zusatz. Wright vermutet, dafs derselbe vielleicht von Dionysius von Tell-Mahrê herrühre. Nöldeke (Z. D. M. G. XXXVI, 1882 S. 689) stimmt ihm darin bei, dafs diese Angabe von einem Spätern herrühre, und hält auch die Vermutung bezüglich des Dionysius für sehr wahr-. scheinlich. Er erklärt das absprechende Urteil über Anastasius daraus, dafs die sehr diphysitische Gesinnung der europäischen Provinzen dem monophysitischen Fürsten gelegentlich diese oder jene Konzession abprefste, welche seinen strengen Glaubensgenossen als Verleugnung der reinen Lehre erscheinen mufste. Indessen die Worte des 101. Kapitels lassen auch eine andre Deutung als möglich zu. Es ist ja gewifs richtig, dafs die Worte unmöglich zu Anastasius' Lebzeiten geschrieben 
sein können; aber müssen sie darum unbedingt dem Josua abgesprochen werden? Die Autorschaft des Dionysius ist doch nur eine unsichere, von Wright mit aller Reserve in einer Note ausgesprochene Vermutung.

Was wir von Josua sicher wissen, ist seine grolse Verehrung für Flavian. Nun wird dieser 512 durch Anastasius abgesetzt, und an seine Stelle tritt der streng monophysitische Severus, der zwar das Henotikon recipiert, aber unter gleichzeitiger Verdammung des Chalcedonense, und mit Johannes von Alexandria und Timotheos von Konstantinopel Gemeinschaft hält. Für Syrien bezeichnet dies das Aufgeben der bisherigen Friedenspolitik des Anastasius; es ist eine Konzession an die strengen Monophysiten, welche jetzt mit derselben fanatischen Intoleranz ihre Herrschaft ausüben, wie später bei der unter Justin eintretenden Reaktion die Orthodoxen. Was ist nun natürlicher, als dafs der milde Josua zur Zeit der drakonischen Mafsregeln Justins und Vitalians, welche die irenischen Henotiker, wie die korrekten Monophysiten gleichmäfsig hart trafen, in dieser Verfolgung aller nichtsynoditisch Gesinnten die Strafe für die Exzesse der Monophysiten strengster Observanz erkannte? Der von ihm einst so gefeierte Kaiser Anastasius trug mit Schuld an der Katastrophe, weil er die Absetzung des friedliebenden und zwischen den Parteien vermittelnden Flavian zum mindesten hatte geschehen lassen; er war eben in seinen letzten Jahren nicht mehr der von Josua bewunderte „allmächtige gläubige Kaiser", sondern wie Salomo in seinem Alter, thöricht geworden. In einem solchen Gedankenzusammenhange scheinen mir die Worte auch als nachträglicher Zusatz des Josua selbst nicht auffällig oder unverständlich; man hat dann nicht nötig, die Worte einem spätern Interpolator zuzuschreiben, wenn man an der auch aus historiographischen Gründen empfehlenswerten Vermutung Gutschmids festhält, dafs die Chronik zwar 5) (oder kurz vorher) verfalst, aber erst nach Anastasius' Tode mit dem nachträglichen Zusatze sei veröffentlicht worden.

Josua steht übrigens mit seiner Gesinnung keineswegs allein. Wir haben dafür das sehr interessante Zeugnis eines spätern entschiedenen Monophysiten, des Jakob von Sarag. Dieser wird gleichmälsig von den Orthodoxen, den Maroniten und den Jakobiten als ihr Heiliger in Anspruch genommen. Dafs er zur strengen Richtung der Monophysiten sich öffentlich bekannte, geht aus den von Martin publizierten Briefen ganz zweifellos hervor. Indessen scheint diese Überzeugung bei ihm nicht immer in der gleichen Stärke vorhanden gewesen zu sein, was Assemani zu dem Versuch veranlafste, ihn als orthodox hinzusteller. Freilich die von ihm angeführten Excerpte zeigen zum Teil eine bedenklich monophysitische Fïrbung; bei anderen allerdings kamn eine ortho- 
doxe Auslegung, wie sie Assemani beliebte, nicht von vornherein als ausgeschlossen bezeichnet werden. Auch zeigt der Briefwechsel mit den Mönchen von Mar Bassus, vor allem die schroffe, fast grobe Antwort auf seine Verdammung des Nestorios, Eutyches, Diodoros, Theodoros und Theodoretos, wie wenig man in streng monophysitischen Kreisen ihm traute. Obschon er seit seiner Jugend, seit 45 Jahren, den Diodor von Tarsos verabscheut zu haben behauptet, verlangen sie und das ist charakteristisch - noch eine ausdrückliche Verdammung von Leos Tomos, vom Konzil von Chalcedon und Annahme des Henotikons u. s. f. Das neue Glaubensbekenntnis, welches Jakob jetzt nicht ohne Verdrufs und leisen Hohn ablegt (Z. D. M. G. XXX, 1876 S. 249) zeigt deutlich, dafs Lazarus und seine Mönche ihn flavianischer Gesinnung für verdächtig hielten. Darum prüft er nun die Frage über das Konzil von Chalcedon „im Lichte der übernatürlichen Wissenschaft" und kommt zu dem Resultate, dafs die Annahme des Henotikons die Verdammung des Konzils in sich schlösse. Von Flavian, „dem Zertrenner Christi", sagt er sich los und bekennt sich zu Severus. Was das Henotikon nur in dunkeln und rätselhaften Worten ausgesagt hat, das hat dieser klar und präzis gefafst. Seine Interpretation des Henotikons, wonach dasselbe "geschickt und ohne Lärm" das Konzil vertilgen soll, ist die allein richtige, von der Synode von Tyros approbierte und von den Alexandrinern von Anfang an vorgetragene Lehre. Sauer genug wird dem Jakob diese „löbliche Unterwerfung“ geworden sein; allein er befand sich den fanatischen Mönchen gegenüber in einer ähnlichen Zwangslage, wie Theodoret in Chalcedon, als er seine Stellung zu Nestorios definieren mulste. Von ihrem Standpunkte aus scheinen auch die Mönche mit ihrem Mifstrauen nicht ganz unrecht gehabt zu haben; denn mit der Thronbesteigung Justins mufs Jakob wieder einen kleinen Frontwechsel gemacht haben; anders vermag ich wenigstens das Faktum nicht $\mathrm{zu}$ erklären, dafs, während sonst in den östlichen Provinzen die monophysitischen Bischöfe massenhaft entsetzt wurden, er selbst 519 auf den Stuhl von Batnae befördert wurde. ') Sein baldiger Tod erlöste ihn von weitern Drangsalierungen, wie sie nach Dionysius von Tell-Mahrê Paulus von Antiochien in chalcedonensischem Eifer bereits gegen ihn begonnen hatte. Bei der leidenschaftlichen Schärfung der Gegensätze war eben für die Mittelpartei kein Platz mehr. Männer wie Josua, welche die Traditionen Flavians und der wahren Henotiker

1) Vgl. auch Abbeloos et Lamy, Gregorii Barhebraei chron. eccles. I p. 924, 925, deren Schlufsfolgerungen ich freilich nicht beizutreten vermag. Die Vermutung, dafs die Mar Bassusbriefe gefälscht seien, ist lediglich eine Verlegenheitsauskúnft. 
aufrecht erhielten, mufsten sich immer mehr vereinsamt fühlen; sie standen auf einem verlornen Posten, oder wenn sie, wie Jakob von Sarûg, der jeweiligen Zeitströmung Konzessionen machten, traf sie nicht mit Unrecht der Vorwurf der Charakterschwäche. Für das Reich war es aber ein unersetzlicher Verlust, dafs die Mittelpartei völlig ausstarb. Denn als Justinian bei seinen zahlreichen kirchenpolitischen Experimenten schliefslich zu der Einsicht kam, dafs seines Oheims unbedingtes Eintreten für das Chalcedonense ein schwerer Mifsgriff gewesen war, und als er demgemäls zur Entrüstung des Occidents das Programm der alten flavianischen Mittelpartei plötzlich für die allein orthodoxe Reichsreligion erklärte, da war es schon zu spät. Die Extremen hatten in Syrien und Ägypten bereits die unbedingte Herrschaft erlangt und verwarfen den neuen Unionsvorschlag mit Hohn. Das folgende Jahrhundert hatte die traurigen Folgen zu tragen.

Jena.

Heinrich Gelzer. 